\title{
Note on Cyclically Interval Edge Colorings of Simple Cycles
}

\author{
Nannan Wang1, Yongqiang Zhao ${ }^{2 *}$ \\ ${ }^{1}$ Institute of Applied Mathematics, Hebei University of Technology, Tianjin, China \\ ${ }^{2}$ School of Mathematics and Information Science, Shijiazhuang University, Shijiazhuang, China \\ Email:981489616@qq.com, ‘yqzhao1970@yahoo.com
}

Received 27 March 2016; accepted 15 July 2016; published 18 July 2016

Copyright (C) 2016 by authors and Scientific Research Publishing Inc.

This work is licensed under the Creative Commons Attribution International License (CC BY).

http://creativecommons.org/licenses/by/4.0/

(c) (i) Open Access

\section{Abstract}

A proper edge $t$-coloring of a graph $G$ is a coloring of its edges with colors $1,2, \cdots, t$ such that all colors are used, and no two adjacent edges receive the same color. A cyclically interval $t$-coloring of a graph $G$ is a proper edge $t$-coloring of $G$ such that for each vertex $x \in V(G)$, either the set of colors used on edges incident to $x$ or the set of colors not used on edges incident to $x$ forms an interval of integers. In this paper, we provide a new proof of the result on the colors in cyclically interval edge colorings of simple cycles which was first proved by Rafayel R. Kamalian in the paper “On a Number of Colors in Cyclically Interval Edge Colorings of Simple Cycles, Open Journal of Discrete Mathematics, 2013, 43-48”.

\section{Keywords}

\section{Edge Coloring, Interval Edge Coloring, Cyclically Interval Edge Coloring}

\section{Introduction}

All graphs considered in this paper are finite undirected simple graphs. For a graph $G$, let $V(G)$ and $E(G)$ denote the sets of vertices and edges of $G$, respectively. For a vertex $x \in V(G)$, let $J_{G}(x)$ and $d_{G}(x)$ denote the subset of $E(G)$ incident with the vertex $x$, and the degree of the vertex $x$ in $G$, respectively. We denote $\Delta(G)$ the maximum degree of vertices of $G$. A simple path with $n \geq 1$ edges is denoted by $P_{n}$. A simple cycle with $n \geq 3$ edges is denoted by $C_{n}$.

For an arbitrary finite set $A$, we denote by $|A|$ the number of elements of $A$. The set of positive integers is denoted by $\mathbb{N}$. An arbitrary nonempty subset of consecutive integers is called an interval. An interval with the

\footnotetext{
"Corresponding author.
} 
minimum element $p$ and the maximum element $q$ is denoted by $[p, q]$. We denote $\diamond[a, b]$ and $\circ[a, b]$ the sets of even and odd integers in $[a, b]$, respectively. An interval $D$ is called a $h$-interval if $|D|=h$.

A function $\alpha: E(G) \rightarrow[1, t]$ is called a proper edge $t$-coloring of a graph $G$, if all colors are used, and no two adjacent edges receive the same color. The minimum value of $t$ for which there exists a proper edge $t$-coloring of a graph $G$ is denoted by $\chi^{\prime}(G)$. If $E_{0} \subseteq E(G)$, and $\alpha$ is a proper edge $t$-coloring of a graph $G$, then let $\left.\alpha\right|_{E_{0}}=\left\{\alpha(e) \mid e \in E_{0}\right\}$. A proper edge $t$-coloring $\alpha$ of a graph $G$ is called an interval $t$-coloring of $G$ if for any $x \in V(G)$, the set $\left.\alpha\right|_{J_{D}(x)}$ is a $d_{G}(x)$-interval. A graph $G$ is interval colorable if it has an interval $t$-coloring for some positive integer $t$. The concept of interval edge coloring of graphs was introduced by Asratian and Kamalian [1]. In [1] [2], the authors showed that if $G$ is interval colorable, then $\chi^{\prime}(G)=\Delta(G)$.

For any $t \in \mathbb{N}$, we denote by $\mathfrak{N}_{t}$ the set of graphs for which there exists an interval $t$-coloring. Let $\mathfrak{N}=\bigcup_{t \geq 1} \mathfrak{N}_{t}$. For any graph $G \in \mathfrak{N}$, the minimum and the maximum values of $t$ for which $G$ has an interval $t$-coloring are denoted by $w(G)$ and $W(G)$, respectively. For a graph $G \in \mathfrak{N}$, let $\theta(G)=\left\{t \mid G \in \mathfrak{N}_{t}\right\}$.

A proper edge $t$-coloring $\alpha$ of a graph $G$ is called a interval cyclic $t$-coloring of $G$, if for any $x \in V(G)$, at least one of the following two conditions holds:

1) $\left.\alpha\right|_{J_{G}(x)}$ is a $d_{G}(x)$-interval,

2) $\left.[1, t] \backslash \alpha\right|_{J_{G}(x)}$ is a $\left(t-d_{G}(x)\right)$-interval.

A graph $G$ is interval cyclically colorable if it has a cyclically interval $t$-coloring for some positive integer $t$. This type of edge coloring under the name of " $\pi$-coloring" was first considered by Kotzig [3], and the concept of cyclically interval edge coloring of graphs was explicitly introduced by de Werra and Solot [4].

For any $t \in \mathbb{N}$, we denote by $\mathfrak{M}_{t}$ the set of graphs for which there exists a interval cyclic $t$-coloring. Let $\mathfrak{M}=\bigcup_{t \geq 1} \mathfrak{M}_{t}$. For any graph $G \in \mathfrak{M}$, the minimum and the maximum values of $t$ for which $G$ has a cyclically interval $t$-coloring are denoted by $w_{c}(G)$ and $W_{c}(G)$ respectively. For a graph $G \in \mathfrak{M}$, let $\Theta(G)=\left\{t \mid G \in \mathfrak{M}_{t}\right\}$.

It is clear that for any $t \in \mathbb{N}, \mathfrak{N}_{t} \subseteq \mathfrak{M}_{t}$ and $\mathfrak{N} \subseteq \mathfrak{M}$. Note that for an arbitrary graph $G, \theta(G) \subseteq \Theta(G)$. It is also clear that for any $G \in \mathfrak{N}$, the following inequality is true:

$$
\Delta(G) \leq \chi^{\prime}(G) \leq w_{c}(G) \leq w(G) \leq W(G) \leq W_{c}(G) \leq|E(G)| .
$$

Let $T$ be a tree. Kamalian [5] [6] showed that $T \in \mathfrak{N}, \theta(T)$ was an interval, and provided the exact values of the parameters $w(T)$ and $W(T)$. Kamalian [7] [8] also proved that $\Theta(T)=\theta(T)$. Some interesting results on cyclically interval $t$-colorings and related topics were obtained in [3] [4] [9]-[14]. For any integer $n \geq 3$, Kamalian [13] proved that $C_{n} \in \mathfrak{M}$, determined the set $\Theta\left(C_{n}\right)$, and provided the following theorem.

Theorem 1 (R. R. Kamalian [13]) For any integers $n \geq 3$ and $t \in[2, n], C_{n} \in \mathfrak{M}_{t}$ if and only if

$$
t \in \begin{cases}\circ[3, n], & \text { if } n \text { is odd; } \\ \diamond\left[\frac{n}{2}+2, n\right] \cup\left[2, \frac{n}{2}+1\right], & \text { if } n \text { is even. }\end{cases}
$$

In this paper, we provide a new proof of the theorem. The terms and concepts that we do not define can be found in [15].

\section{Main Result}

Proof of Theorem 1. Suppose that, in clockwise order along the cycle $C_{n}$, the vertices of $C_{n}$ are $v_{1}, v_{2}, \cdots, v_{n}$ and the edges of $C_{n}$ are $e_{1}, e_{2}, \cdots, e_{n}$, where $e_{i}=v_{i} v_{i+1}$ for $i=1,2, \cdots, n$, and $v_{n+1}=v_{1}$. Since $\left|E\left(C_{n}\right)\right|=n$ and

$$
\chi^{\prime}\left(C_{n}\right)= \begin{cases}2, & \text { if } n \text { is even; } \\ 3, & \text { if } n \text { is odd }\end{cases}
$$

We know that if $t>n$ or

$$
t< \begin{cases}2, & \text { if } n \text { is even; } \\ 3, & \text { if } n \text { is odd }\end{cases}
$$


then $C_{n} \notin \mathfrak{M}_{t}$.

First we prove that if $n \geq 3$ and

$$
t \in \begin{cases}\circ[3, n], & \text { if } n \text { is odd } \\ \diamond\left[\frac{n}{2}+2, n\right] \cup\left[2, \frac{n}{2}+1\right], & \text { if } n \text { is even, }\end{cases}
$$

then $C_{n} \in \mathfrak{M}_{t}$.

Case $1 . n$ is odd.

For any $t \in \circ[3, n]$, let

$$
\alpha\left(e_{i}\right)= \begin{cases}i, & i \in[1, t] \\ 1, & i \in \diamond[t+1, n] ; \\ 2, & i \in \circ[t+1, n] .\end{cases}
$$

It is easy to check that $\alpha$ is a cyclically interval $t$-coloring of $C_{n}$.

Case 2. $n$ is even.

For any $t \in \diamond[2, n]$, let

$$
\alpha\left(e_{i}\right)= \begin{cases}i, & i \in[1, t], \\ 1, & i \in \circ[t+1, n] \\ 2, & i \in \diamond[t+1, n] .\end{cases}
$$

If $t=\frac{n}{2}+1$ is odd, then let

$$
\alpha\left(e_{i}\right)= \begin{cases}i, & i \in[1, t] ; \\ n-i+2, & i \in[t+1, n] .\end{cases}
$$

For any $t \in \circ\left[3, \frac{n}{2}\right]$, let

$$
\alpha\left(e_{i}\right)= \begin{cases}i, & i \in[1, t] \\ i-t, & i \in[t+1,2 t] ; \\ 1, & i \in \circ[2 t+1, n] ; \\ 2, & i \in \diamond[2 t+1, n] .\end{cases}
$$

It is easy to check that, in each case, $\alpha$ is a cyclically interval $t$-coloring of $C_{n}$.

Now let us prove that if $n \geq 3, t \in[2, n]$ and $C_{n} \in \mathfrak{M}_{t}$, then

$$
t \in \begin{cases}\circ[3, n], & \text { if } n \text { is odd; } \\ \diamond\left[\frac{n}{2}+2, n\right] \cup\left[2, \frac{n}{2}+1\right], & \text { if } n \text { is even. }\end{cases}
$$

By contradiction. Suppose that there are $n_{0} \in \mathbb{N}, n_{0} \geq 3, t_{0} \in\left[2, n_{0}\right]$ and

$$
t_{0} \notin \begin{cases}\circ[3, n], & \text { if } n_{0} \text { is odd; } \\ \diamond\left[\frac{n}{2}+2, n\right] \cup\left[2, \frac{n}{2}+1\right], & \text { if } n_{0} \text { is even, }\end{cases}
$$

such that $C_{n_{0}}$ has a cyclically interval $t_{0}$-coloring $\alpha$.

Case 1. $n_{0}$ is odd.

Clearly, $t_{0} \in \diamond\left[2, n_{0}-1\right]$. Let $e_{s}$ and $e_{t}$ be two edges of $C_{n_{0}}$ such that $\alpha\left(e_{s}\right)=1$ and $\alpha\left(e_{t}\right)=t_{0}$. Without loss of generality, we may assume $s<t$. Let $L_{1}$ be the subgraph induced by $\left\{e_{i} \mid s \leq i \leq t\right\}$, and $L_{2}$ be the subgraph induced by $\left\{e_{j} \mid j \leq s\right.$ or $\left.j \geq t\right\}$, respectively. Since $t_{0}$ is even and $\alpha$ is a cyclically interval 
$t_{0}$-coloring of $C_{n_{0}}$, then $\left|E\left(L_{1}\right)\right|$ and $\left|E\left(L_{2}\right)\right|$ are all even. So we have that $n_{0}$ is even, a contradiction.

Case 2. $n_{0}$ is even.

Let $H$ be the graph removing from the graph $C_{n_{0}}$ the edges with the colors except 1 and $t_{0}$, and $H_{0}$ the graph removing from the graph $H$ all its isolated vertices.

Case 2.1. $H_{0}$ is connected.

Let $F$ be the subgraph of $C_{n_{0}}$ induced by $E\left(C_{n_{0}}\right) \backslash E\left(H_{0}\right) \cup\left\{e^{\prime}, e^{\prime \prime}\right\}$, where $e^{\prime}$ and $e^{\prime \prime}$ are the two pendant edges of $H_{0}$.

Clearly, $t_{0} \in \circ\left[\frac{n_{0}}{2}+2, n_{0}-1\right]$. If $\left|E\left(H_{0}\right)\right|$ is odd, then $\alpha\left(e^{\prime}\right)=\alpha\left(e^{\prime \prime}\right)$. Since $\alpha$ is a cyclically interval $t_{0}$ coloring of $C_{n_{0}}$, then $\left.\alpha\right|_{E(F)}$ is a interval $\left(t_{0}-1\right)$-coloring with $\alpha\left(e^{\prime}\right)=\alpha\left(e^{\prime \prime}\right)$. So we have $n_{0}>|E(F)| \geq 2 t_{0}-3 \geq n_{0}+1$, a contradiction.

If $\left|E\left(H_{0}\right)\right|$ is even, then $\alpha\left(e^{\prime}\right) \neq \alpha\left(e^{\prime \prime}\right)$. Since $\alpha$ is a cyclically interval $t_{0}$-coloring of $C_{n_{0}}$, then $\left.\alpha\right|_{E(F)}$ is a interval $t_{0}$-coloring. So we know that $|E(F)|$ is odd, and then $n_{0}=\left|E\left(H_{0}\right)\right|+|E(F)|-2$ is odd, a contradiction.

Case 2.2. $H_{0}$ is a graph with $m$ connected components, $m \geq 2$.

Suppose that, in clockwise order along the cycle $C_{n}$, the $m$ connected components of $H_{0}$ are $H_{1}, H_{2}, \cdots, H_{m}$. Without loss of generality, we may also assume that $v_{1}, v_{2} \in V\left(H_{1}\right)$ and $v_{n_{0}} \notin V\left(H_{1}\right)$.

Clearly, $t_{0} \in \circ\left[\frac{n_{0}}{2}+2, n_{0}-1\right]$ and $\min \left\{i \mid e_{i} \in E\left(H_{1}\right)\right\}=1$. Let $r_{1}=\max \left\{i \mid e_{i} \in E\left(H_{1}\right)\right\}$,

$r_{2}=\min \left\{i \mid e_{i} \in E\left(H_{2}\right)\right\}$ and $r_{3}=\max \left\{i \mid e_{i} \in E\left(H_{m}\right)\right\}$. Let $L_{3}$ be the subgraph induced by $\left\{e_{i} \mid r_{1} \leq i \leq r_{2}\right\}$, and $L_{4}$ be the subgraph induced by $\left\{e_{j} \mid j=1\right.$ or $\left.j \geq r_{3}\right\}$, respectively. Let $\alpha\left(e_{r_{1}}\right)=\alpha\left(e_{r_{2}}\right)$ or $\alpha\left(e_{r_{3}}\right)=\alpha\left(e_{1}\right)$, say $\alpha\left(e_{r_{1}}\right)=\alpha\left(e_{r_{2}}\right)$. Since $\alpha$ is a cyclically interval $t_{0}$-coloring of $C_{n_{0}}$, then $\left.\alpha\right|_{E\left(L_{3}\right)}$ is a interval $\left(t_{0}-1\right)$ coloring with $\alpha\left(e_{\eta_{1}}\right)=\alpha\left(e_{r_{2}}\right)$. So we have $n_{0}>\left|E\left(L_{3}\right)\right| \geq 2 t_{0}-3 \geq n_{0}+1$, a contradiction.

Now let $\alpha\left(e_{r_{1}}\right) \neq \alpha\left(e_{r_{2}}\right)$ and $\alpha\left(e_{r_{3}}\right) \neq \alpha\left(e_{1}\right)$. Since $\alpha$ is a cyclically interval $t_{0}$-coloring of $C_{n_{0}}$, then $\left.\alpha\right|_{E\left(L_{3}\right)}$ and $\left.\alpha\right|_{E\left(L_{4}\right)}$ are all interval $t_{0}$-coloring. So we have $n_{0}>\left|E\left(L_{3}\right)\right|+\left|E\left(L_{4}\right)\right|-2 \geq 2 t_{0}-2 \geq n_{0}+2$, a contradiction.

\section{Acknowledgements}

We thank the editor and the referee for their valuable comments. Research of Y. Zhao is funded in part by the Natural Science Foundation of Hebei Province of China under Grant No. A2015106045, and in part by the Institute of Applied Mathematics of Shijiazhuang University.

\section{References}

[1] Asratian, A.S. and Kamalian, R.R. (1987) Interval Colorings of Edges of a Multigraph. Applied Mathematics, 5, 25-34. (In Russian)

[2] Asratian, A.S. and Kamalian, R.R. (1994) Investigation on Interval Edge-Colorings of Graphs. Journal of Combinatorial Theory, Series B, 62, 34-43. http://dx.doi.org/10.1006/jctb.1994.1053

[3] Kotzig, A. (1979) 1-Factorizations of Cartesian Products of Regular Graphs. Journal of Graph Theory, 3, 23-34. http://dx.doi.org/10.1002/jgt.3190030104

[4] Werra, D. and Solot, Ph. (1989) Compact Cylindrical Chromatic Scheduling. ORWP 89/10, Ecole Polytechnique Fédérale de Lausanne.

[5] Kamalian, R.R. (1990) Interval Edge Colorings of Graphs. PhD Dissertation, the Institute of Mathematics of the Siberian Branch of the Academy of Sciences of USSR, Novosibirsk. (In Russian)

[6] Kamalian, R.R. (1989) Interval Coloring of Complete Bipartite Graphs and Trees. Preprint of the Computing Centre of the Academy of Sciences of Armenia. (In Russian)

[7] Kamalian, R.R. (2010) On a Number of Colors in Cyclically Interval Edge Colorings of Trees. Research Report LiTHMAT-R-2010/09-SE, Linkoping University. 
[8] Kamalian, R.R. (2012) On Cyclically-Interval Edge Colorings of Trees. Buletinul Academiei de Stiinte a Republicii Moldova Matematica, 68, 50-58.

[9] Bartholdi, J.J., Orlin, J.B. and Ratliff, H.D. (1980) Cyclic Scheduling via Integer Programs with Circular Ones. Operations Research, 28, 1074-1085. http://dx.doi.org/10.1287/opre.28.5.1074

[10] Dauscha, W., Modrow, H.D. and Neumann, A. (1985) On Cyclic Sequence Type for Constructing Cyclic Schedules. Zeitschrift für Operations Research, 29, 1-30. http://dx.doi.org/10.1007/bf01920492

[11] Werra, D., Mahadev, N.V.R. and Solot, P. (1989) Periodic Compact Scheduling. ORWP 89/18, Ecole Polytechnique Fédérale de Lausanne.

[12] Kamalian, R.R. (2007) On Cyclically Continues Edge Colorings of Simple Cycles. Proceedings of the Computer Science and Information Technologies Conference, Yerevan, 24-28 September 2007, 79-80. (In Russian)

[13] Kamalian, R.R. (2013) On a Number of Colors in Cyclically Interval Edge Colorings of Simple Cycles. Open Journal of Discrete Mathematics, 3, 43-48. http://dx.doi.org/10.4236/ojdm.2013.31009

[14] Petrosyan, P.A. and Mkhitaryan, S.T. (2014) Interval Cyclic Edge-Colorings of Graphs. http://arxiv.org/abs/1411.0290v1

[15] West, D.B. (1996) Introduction to Graph Theory. Prentice Hall, Upper Saddle River.

\section{Submit or recommend next manuscript to SCIRP and we will provide best service for you:}

Accepting pre-submission inquiries through Email, Facebook, Linkedin, Twitter, etc A wide selection of journals (inclusive of 9 subjects, more than 200 journals)

Providing a 24-hour high-quality service

User-friendly online submission system

Fair and swift peer-review system

Efficient typesetting and proofreading procedure

Display of the result of downloads and visits, as well as the number of cited articles

Maximum dissemination of your research work

Submit your manuscript at: http://papersubmission.scirp.org/ 